\title{
Orbital solutions derived from radial velocities and time delays for four Kepler systems with A/F-type (candidate) hybrid pulsators ${ }^{\star}$
}

\author{
P. Lampens ${ }^{1}$, L. Vermeylen ${ }^{1}$, Y. Frémat ${ }^{1}$, Á. Sódor ${ }^{2}$, M. Skarka ${ }^{3,4}$, A. Samadi-Ghadim ${ }^{5}$, Zs. Bognár ${ }^{2,6}$, H. Lehmann ${ }^{7}$, \\ P. De Cat ${ }^{1}$, A. Goswami ${ }^{8}$, and L. Dumortier ${ }^{1}$
}

1 Koninklijke Sterrenwacht van België, Ringlaan 3, 1180 Brussel, Belgium e-mail: patricia.lampens@oma.be

2 Konkoly Observatory, Research Centre for Astronomy and Earth Sciences \& MTA CSFK Lendület Near-Field Cosmology Research Group, Konkoly Thege M. u. 15-17, H-1121, Budapest, Hungary

3 Astronomical Institute, Czech Academy of Sciences, Fričova 298, CZ-25165 Ondřejov, Czech Republic

4 Department of Theoretical Physics and Astrophysics, Masaryk Univerzity, Kotlářská 2, CZ-61137 Brno, Czech Republic

5 Núcleo de Astronomía, Faculdad de Ingeniería y Ciencias, Universidad Diego Portales, Av. Ejército Libertador 441, Santiago, Chile

${ }^{6}$ ELTE Eötvös Loránd University, Institute of Physics, Pázmány Péter sétány 1/A, H-1171, Budapest, Hungary

7 Thüringer Landessternwarte, Tautenburg, Germany

8 Indian Institute of Astrophysics, II Block, Koramangala, Bangalore 560 034, India

Received...; accepted ...

\section{ABSTRACT}

\begin{abstract}
Context. The presence of A/F-type Kepler hybrid stars extending across the entire $\delta$ Sct- $\gamma$ Dor instability strips and beyond remains largely unexplained. In order to better understand these particular stars, we performed a multi-epoch spectroscopic study of a sample of 49 candidate A/F-type hybrid stars and one cool(er) hybrid object detected by the Kepler mission. We determined a lower limit of $27 \%$ for the multiplicity fraction. For six spectroscopic systems, we also reported long-term variations of the time delays. For four systems, the time delay variations are fully coherent with those of the radial velocities (RVs) and can be attributed to orbital motion. Aims. We aim to improve the orbital solutions for those spectroscopic systems with long orbital periods (order of 4-6 years) among the Kepler hybrid stars that we continued to observe.

Methods. The orbits are computed based on a simultaneous modelling of the RVs obtained with high-resolution spectrographs and the photometric time delays (TDs) derived from time-dependent frequency analyses of the Kepler light curves.

Results. We refined the orbital solutions of four spectroscopic systems with A/F-type Kepler hybrid component stars: KIC 4480321, 5219533, 8975515 and KIC 9775454. Simultaneous modelling of both data types analysed together enabled us to improve the orbital solutions (all), obtain more robust and accurate information on the mass ratio (some for the first time), and identify the component with the short-period $\delta$ Sct-type pulsations (all). The information gained is maximized when one of the components, generally the one exhibiting the $\delta$ Sct-type pulsations, is the fast(er) spinning component. In several cases, we were also able to derive new constraints for the minimum component masses. From a search for regular frequency patterns in the high-frequency regime of the Fourier transforms of each system, we found no evidence of tidal splitting among the triple systems with close (inner) companions. However, some systems exhibit frequency spacings which can be explained by the mechanism of rotational splitting.
\end{abstract}

Key words. binaries: spectroscopic - stars: variables: delta Scuti - asteroseismology - techniques: radial velocities - techniques: photometric

\section{Introduction}

In an effort to understand and unravel the enigma of the low frequencies in the brighter A/F-type candidate hybrid pulsators of the Kepler mission (Grigahcène et al. 2010, Uytterhoeven et al. 2011; Balona et al. 2015, Bowman |2017), we started a radial-velocity monitoring campaign with high-resolution échelle spectrographs located in various European observatories. Our goal is to characterize the spectroscopic variability of an unbiased sample of Kepler hybrid ( $\gamma$ Dor $-\delta$ Sct) pulsators. This programme was initiated during the middle of 2013. We collected multi-epoch observations (4 - 6 times at least) in order

\footnotetext{
^ This study is based on spectra obtained with the HERMES échelle spectrograph installed at the Mercator telescope, operated by the IvS, KULeuven, funded by the Flemish Community and located at the Observatorio del Roque de los Muchachos, La Palma, Spain of the Instituto de Astrofísica de Canarias.
}

to detect binarity or multiplicity at the different time scales, with orbital periods ranging from a few days to several years, and to establish a meaningful classification. We determined the epoch radial velocities, projected rotational velocities, new or improved atmospheric stellar properties (i.e. $T_{\text {eff }}, \log g, v \sin i$ ), and we provided a classification in terms of multiplicity, pulsation and/or (fast) rotation for all our targets on the basis of the shapes of the cross-correlation functions and their radial velocities as a function of time (Lampens et al.2018, from hereon Paper I).

In this first study, we followed a sample of 49 candidate hybrid stars and one much cooler hybrid object identified by Uytterhoeven et al. (2011). We found a significant rate of short- and long-period binary as well as multiple systems as we detected 10 spectroscopic systems in total, i.e. 4 double-lined (SB2) systems, 3 triple-lined (SB3) systems, 4 single-lined (SB1) systems (only 3 belong to the A/F-class), and 3 objects 
with long-term radial-velocity variations (VAR). We determined the orbital solutions of seven systems. For two hierarchical triple systems, we also proposed a preliminary solution for the outer orbit. Using the classification results, we provided a lower limit of the fraction of A/F-type hybrid pulsators which belong to spectroscopic binary and multiple systems. Including the known Kepler eclipsing binary KIC 11180361 (KOI-971), we derived a minimum multiplicity fraction of $27 \%$. Two other hybrid targets have a possible companion or shell (CMP). If we count these two targets, we obtain an overall multiplicity fraction of about $30 \%$ among these candidate hybrid pulsators.

Among the new spectroscopic systems, we identified four cases for which analysis of the Kepler time delays (TDs) (mostly derived from the short-period pulsation frequencies) with the radial velocities (RVs) enables us to derive improved orbital elements, accurate mass ratios as well as a (most) probable identification of the pulsating component. The goal of this paper is to determine orbital solutions for these systems as accurately as possible based on the results of a unified modelling by combining the various data types into a single, simultaneous analysis. In the present study, we will make usage of the radial velocities acquired with the échelle spectrograph HERMES equipping the 1.2-m Mercator telescope, La Palma, Spain (Raskin et al.2011) available from Paper I (see table C.2). Furthermore, we reported variable TDs in nine cases, in particular for the spectroscopic systems KIC 4480321, 5219533, 8975515, and KIC 9775454 (Paper I, fig. 24). For all four systems, our spectroscopic observations were acquired until late 2019. For KIC 9775454, we included the measurements obtained in 2018 with the high-resolution échelle spectrograph HESP (R $=60,000)$ mounted at the Himalayan Chandrasekhar telescope (HCT), operated by the IIA, Bangalore, India 1 Consequently, all RV data of all four systems and their components were updated to the latest possible date. First, we present the current status of each individual system (Sect. 2). In Sect. 3, we introduce the methodology for computing the refined orbits. Sect. 4 provides the orbital solutions using simultaneous modelling. In Sect.5, we present and discuss the distributions of the frequency spacings in the high-frequency region of the Fourier transforms of each system. Finally, we present our conclusions for all the treated systems (Sect. 6).

\section{Presentation of the systems}

We present a summary of useful high-resolution spectra for each of the four systems listed in Table 1 . In addition to the total number of spectra available for each system, we also mention the spectrograph and the time basis. The revised orbital solutions (Sect. 4) are thus based on the combination of updated sets of component RVs and the time delays (TDs) previously derived from time-dependent frequency analyses of the oscillations detected in the Kepler light curves (Paper I), providing us with a total time basis longer than eight years for each case.

\subsection{KIC 4480321}

KIC 4480321 (HD 225479, V=10.3, A9 Vwkmet) is a hybrid variable star revealed by Uytterhoeven et al. (2011), with frequencies in the ranges $[0.2,5.0] \mathrm{d}^{-1}$ and $[5.1,61.2] \mathrm{d}^{-1}$ and

\footnotetext{
$\overline{1} \mathrm{https} / / /$ www.iiap.res.in/hesp
}

Table 1: List of targets and general properties of the RV data.

\begin{tabular}{cccccc}
\hline \hline KIC ID & Nr & $\begin{array}{c}\text { BJD start } \\
2,450,000 .+\end{array}$ & $\begin{array}{c}\text { BJD end } \\
2,450,000 .+\end{array}$ & $\begin{array}{c}\text { Time range } \\
\text { (from/to) }\end{array}$ & Instr| ${ }^{2}$ \\
\hline 4480321 & 61 & 5820.4499 & 8676.4431 & $15 / 09 / 2011-11 / 07 / 2019$ & $\mathrm{H} 1$ \\
5219533 & 52 & 5372.7083 & 8792.3578 & $25 / 06 / 2010-04 / 11 / 2019$ & $\mathrm{H} 1$ \\
8975515 & 31 & 5345.7088 & 8792.3686 & $29 / 05 / 2010-04 / 11 / 2019$ & $\mathrm{H} 1$ \\
9775454 & 26 & 5345.7325 & 8794.3056 & $29 / 05 / 2010-06 / 11 / 2019$ & $\mathrm{H} 1$ \\
9775454 & 2 & 8231.4431 & 8232.3771 & $22 / 04 / 2018-23 / 04 / 2018$ & $\mathrm{H} 2$ \\
\hline
\end{tabular}

Notes. ${ }^{(2)}$ H1 stands for 'HERMES'. H2 stands for 'HESP'.

a most dominant frequency of $0.710 \mathrm{~d}^{-1}$ in the $\gamma$ Dor region. The star was also reported as rotationally variable (Nielsen et al. 2013), with g-mode multiplets split by rotation. The period spacings in the $\gamma$ Dor region were obtained by Li et al. (2019). This star was classified as an SB3 from our multi-epoch study. It is a hierarchical system, with a twin-like inner binary consisting of early F-type stars orbiting a slightly more luminous and more rapidly rotating A-type companion (we used the basic model of an A5-star, $v \sin i=160 \mathrm{~km} \mathrm{~s}^{-1}$ with $2 \mathrm{~F} 0$-stars, $v \sin i=$ $10 \mathrm{~km} \mathrm{~s}^{-1}$, Paper I). We derived an orbital solution for the inner pair with a 9.1659 d period, and an outer orbital solution with an estimated $2280 \mathrm{~d}$ period, and reported the existence of variable TDs in excellent agreement with the proposed solution for the outer orbit. We determined the component effective temperatures $T_{\text {eff } 1}=7900 \pm 100 \mathrm{~K}$ and $T_{\text {eff } 2}$ and $T_{\text {eff } 3}$ ranging between 6300 and $6900 \mathrm{~K}$ (the latter components have almost similar temperatures), assuming $\log g=4 \mathrm{dex}$ (cgs) (Paper I, Sect. 6.3). Murphy et al. (2018, Appendix C3) reported $P=$ $2270 \pm 60 \mathrm{~d}$ for the outer orbit from a modelling of Lampens et al. (2018)'s RV measurements in combination with their time delays.

\subsection{KIC 5219533}

KIC 5219533 (HD 226766, BU 1474 B, V=9.2, A2-A8, Renson \& Manfroid 2009) is the visual companion of BU $1474 \mathrm{~A}$ at an angular separation of 65" (HD 189178, V=5.44, B5 He weak (Renson \& Manfroid 2009); also a spectroscopic binary). This star was classified as a Kepler hybrid variable star by Uytterhoeven et al. (2011), with frequencies in the ranges [0.3, 4.6] $\mathrm{d}^{-1}$ and $[5.4,29.9] \mathrm{d}^{-1}$, and a dominant frequency of 10.285 $\mathrm{d}^{-1}$ (Uytterhoeven et al. 2011, Table 3). It was recognized as a new SB3 based on the multi-epoch spectra. The system is hierarchical, consisting of an inner pair with two nearly identical stars of spectral type near A5 and a more rapidly rotating outer component of a slightly cooler type. For both components of the inner pair, the mean effective temperatures of $T_{\text {eff } 1}=8300 \pm 100$ $\mathrm{K}$ and $T_{\text {eff } 2}=8200 \pm 100 \mathrm{~K}$ and projected rotational velocities of $10 \mathrm{~km} \mathrm{~s}^{-1}$ were obtained coupled to the light factor $1_{1}=$ $0.53 \pm 0.02$, assuming $\log g=4 \operatorname{dex}(\mathrm{cgs})$ (Paper I, Sect. 6.4). Component $\mathrm{C}$ has $v \sin i$ equal to $115 \mathrm{~km} \mathrm{~s}^{-1}$ Uytterhoeven et al. (2011). An orbital solution with $P_{\text {orb }}=31.9181 \mathrm{~d}$ was derived for the inner pair, while a tentative outer orbit was proposed with $P_{\text {orb }} \sim 1600 \mathrm{~d}$. Later on, Murphy et al. (2018. Appendix C4) studied this system using the phase modulation (PM) method (Murphy et al. 2014). They derived a very high mass function and proposed that the third body is a $\delta$ Sct star with an orbital period longer than $1500 \mathrm{~d}$. Simultaneously with Lampens et al. (2018), Catanzaro et al. (2019a b) independently observed the system (from 2014 to 2018) with the aim to 
characterize the orbits, and to perform a chemical analysis of its components. They proposed the orbital parameters based on their and Lampens et al. (2018)'s RVs $\left(P_{1}=31.9187 \mathrm{~d}, e_{1}=\right.$ $0.28, q_{1}=1.03$ and $\left.P_{2}=1615 \mathrm{~d}, e_{2}=0.54, f\left(M_{1,2}\right)=0.18\right)$ which confirm the findings of Paper I. They also derived the atmospheric properties $T_{\text {eff }}, \log g$, and $v \sin i$ based on 2 spectra (obtained at max RV separation) and chemical abundances based on one spectrum $(\max \mathrm{S} / \mathrm{N})$, first for two components, next with the third one included. However, they were unable to derive the chemical composition of component $\mathrm{C}$ (except for $\mathrm{Mg}$ II). They concluded that both components of the close pair are twin Am stars (with underabundances of $\mathrm{C}, \mathrm{O}, \mathrm{Mg}, \mathrm{Ca}$ and $\mathrm{Sc}$ and overabundances of $\mathrm{Na}, \mathrm{Fe}-$ peak elements). From the simple assumption that all the components have equal mass, they suggested that the orbits might be coplanar. All previous studies explicitly mention that more data for this multiple system are needed.

\subsection{KIC 8975515}

KIC 8975515 (HD 188538, V=9.5, A6 V:) was classified as a Kepler hybrid star by Uytterhoeven et al. (2011). The star shows frequencies in the ranges $[0.3-4.7] \mathrm{d}^{-1}$ and $[5.3-25.8] \mathrm{d}^{-1}$ of the $\gamma$ Dor and the $\delta$ Sct pulsation regimes respectively, with the dominant frequency of $13.97 \mathrm{~d}^{-1}$ (Uytterhoeven et al. 2011, Table 3). Therefore, we included it among our sample of A/Ftype candidate hybrid stars to be monitored spectroscopically where it was recognized as a new SB2 from the multi-epoch observations. The system consists of two A-type stars of similar temperature but with dissimilar projected rotational velocities, forming an eccentric system $(e=0.409 \pm 0.015)$. From a detailed spectrum fitting using the code SYNSPEC (Hubeny \& Lanz 1995) and the ATLAS-9 atmosphere models (Castelli \& Kurucz 2003) in order to build a suitable composite model in the spectral range [500-520] $\mathrm{nm}$, the mean effective temperatures $T_{\text {eff } 1}=7440 \pm 20 \mathrm{~K}$ and $T_{\text {eff } 2}=7380 \pm 21 \mathrm{~K}$ with the light factor $l_{1}$ of $0.65 \pm 0.03$ (component A), assuming $\log g=4 \operatorname{dex}(\mathrm{cgs})$, were obtained. The mean projected rotation velocities were consistently determined as $162 \pm 2$ and $32 \pm 1 \mathrm{~km} \mathrm{~s}^{-1}$ (Paper I, Sect. 6.12). Lampens et al. (2018) proposed a preliminary orbit with a period of the order of $1600 \mathrm{~d}$, in line with the TD curve, while Murphy et al. (2018, Appendix C12) obtained a solution with a shorter period of $1090 \mathrm{~d}$, using the published RVs in combination with their time delays (TDs). They furthermore assumed that the narrow-lined component is the $\delta$ Scuti pulsator and reported a slight aperiodicity in their derived TDs.

An extensive study of its pulsational properties was recently published by Samadi-Ghadim et al. (2020). They concluded that both components are pulsating, the fast-rotating component is a pulsating hybrid star also showing retrograde $r$ modes, while the more slowly-rotating component is a $\delta$ Scuti pulsator.

\subsection{KIC 9775454}

KIC 9775454 (HD 185115, V=8.2, F1 Vs) is a candidate hybrid variable star from Uytterhoeven et al. (2011), with frequencies in the ranges $[0.2,4.6] \mathrm{d}^{-1}$ and $[14.7,14.9] \mathrm{d}^{-1}$ and a dominant frequency of $4.161 \mathrm{~d}^{-1}$. It has a Gaia DR2 RV of $-20.22 \pm 1.02$ $\mathrm{km} \mathrm{s}^{-1}$ (Gaia Collaboration 2018). The atmospheric parameters derived from high-resolution spectroscopy are $T_{\text {eff }}=7287 \mathrm{~K}$ and $\log g=4.25 \mathrm{dex}$ (cgs) with a projected rotation velocity of $v \sin i=65 \mathrm{~km} \mathrm{~s}^{-1}$ (Paper I, Table C.1). Lampens et al. (2018) classified this object as a long-term SB1 of unknown orbital period. The cross-correlation function (CCF) of the composite spectrum shows a broad-lined component blended with a narrow-lined component. Murphy et al. (2018, Appendix C14) proposed an orbit with $P_{\text {orb }}=1686 \pm 13 \mathrm{~d}$ and $e=0.23$ \pm 0.02 by combining the published RVs with their TDs. In this case, the secondary component contributes only weakly to the composite spectrum. Nevertheless, we were able to obtain some individual RVs for this (much) cooler component (cf. Sect. 4.4).

\section{Methodology}

The orbital motion of a binary system causes a periodic fluctuation of the light path and its associated travel time with respect to the system's centre of mass. On the other hand, light from a source in motion undergoes a (Doppler) shift of its frequency. In the frequency domain (for a time base $T \gg P_{\text {orb }}$ ), this phenomenon corresponds to a frequency modulation (FM) which can be detected as a frequency multiplet whose exact spacing is the orbital frequency (Shibahashi \& Kurtz 2012). In the time domain (for $T \ll P_{\text {orb }}$ ), this effect corresponds to a modulation of the phase (PM) with the orbital period, whose amplitude depends on the mass of the (unseen) companion and the observed frequency (Murphy et al.|2014). Hence, if one of the components is a pulsating star, the change in the light travel time introduces periodic phase shifts of each individual (pulsation) frequency. By converting the phase shift into a time delay, the frequency dependency is removed. Therefore, if the star is a multiperiodic pulsator, all the pulsation frequencies will experience the same time delay.

Murphy et al. (2014) described the principle of obtaining time delays from observed phase shifts of the pulsations and applied the PM method unto various cases of Kepler pulsating stars in (non-eclipsing) binary systems, while Murphy \& Shibahashi (2015) provided an analytical method for solving the orbit, also in highly eccentric cases. This method is in essence similar to the search for variability in the (O-C)'s, the residuals in the times of specific phases in the light curve of periodically pulsating stars which are members of a binary system (e.g. Moffett et al. 1988, Fauvaud et al. 2010). Murphy et al. (2016) presented examples of orbital analyses combining radial velocities (RVs) with photometric time delays (TDs), and discussed some of the limitations and advantages. In this study, we developed and applied our own code for a simultaneous least-squares fitting of our updated RVs and the TDs based on the Kepler data. Both quantities are functions of the same orbital parameters: the time delay corresponds to the light travel time and the radial velocity is the time derivative of the position along the orbital path projected in the observer's direction. We consider the following equations as our model:

$\mathrm{TD}(t)=\frac{a \sin i}{c} \frac{1-e^{2}}{1+e \cos (v(t))} \sin (v(t)+\omega)$

$V(t)=2 \pi \frac{a \sin i}{P \sqrt{1-e^{2}}}(\cos (v(t)+\omega)+e \cos \omega)+V_{\gamma}$,

where the true anomaly $v$ is a function of $t, T_{0}, P$ and $e, T_{0}$ is the time of periastron passage, $P$ is the orbital period, $a \sin i$ is the projected semi-major axis, $e$ is the eccentricity, $\omega$ is the 
longitude of the periastron, $V_{\gamma}$ is the systemic velocity and $\mathrm{c}$ is the speed of light. Equ. 1 is given by Irwin (1952) (see Pribulla et al. 2005). We removed a linear trend from the original TDs computed with Equ. 1 (Murphy et al. 2016) in order to convert the pulsation frequencies to their intrinsic values, i.e. unaffected by the variable light travel time of the pulsating component. This correction is necessary since the frequencies used for computing the TDs may be biased by the (uneven) orbital phase coverage of the Kepler data, thereby introducing a bias in the TDs themselves (as in the (O-C) curves).

In Paper I, we computed the time-dependent phases for up to 20 frequencies of highest $S / N$ (generally the ones located in the $p$-mode regime) for all 49 targets of our sample. We thus uncovered nine objects for which correlated variations of comparable amplitude in the TDs were observed. In six systems, we could attribute the TD variations to orbital motion of a binary or multiple system. By analysing the RV and the TD data together, we are able to refine the orbital solutions and obtain stronger constraints on (some of) the orbital parameters and their derived parameters. Since the TDs are generally determined from the high(er) frequencies, they only provide information on the origin of the $\delta$ Scuti-type pulsations associated to these frequencies. However, it is much harder to identify the origin of the low(er) frequencies ( $\gamma$ Doradus-type) due to their longer periods. In the next sections, we will analyse each of the four systems just described and highlight the gains that may be obtained from such modelling.

\section{Orbital solutions from combined modelling}

\subsection{The triple system KIC 4480321}

KIC 4480321 is a triple system (SB3) whose orbital periods are estimated to be 9.166 and $\sim 2280 \mathrm{~d}$ (Sect. 2.1). We computed the phase shifts and their associated TDs for frequencies of highest $S / N$ and evidenced the presence of long-term variations which are coherent with the long-term RV variability (Paper I, fig. 24). We remark that the TDs were corrected by removing a linear trend from the original data (as everywhere). We then performed a simultaneous least-squares fitting of three updated component RVs and the mean TDs with their respective uncertainties. The best fit in the sense of min $\chi^{2}$ was obtained when we associated the TDs with the RVs of the fast spinning, more luminous A-type star (component C). Fig. 11 illustrates the quality of the fit showing the outer orbital solution together with both data types. The TDs and the RVs are overlaid with the AB-C orbital solution. Note that the TD curve mimics the RV curve but with an apparently larger eccentricity and a $90^{\circ}$ shift in periastron longitude (Irwin 1952). The revised orbital parameters of the coupled solutions are listed in Table 2. Compared to Paper I, we see that the orbital solution of the A-B system is robust and well-determined while the outer orbital solution changed profoundly. The orbital period is longer $\left(P_{\text {orb }} \sim 2380 \mathrm{~d}\right)$ and better determined than before (see also Murphy et al. 2018), while the eccentricity and the semi-axis major increased substantially. Thus, the minimum component mass $M_{\mathrm{C}} \sin ^{3} i_{\text {out }}$ increased to $1.60 \pm 0.05 \mathrm{M}_{\odot}$. The strongest improvement concerns the accuracy and robustness of the outer orbital solution. This is due to two things: (a) by combining both data types, we dispose of a time base longer than eight years and (b) the TDs concern the fast-rotating component $\mathrm{C}$ for which the RVs show the largest scatter. We therefore were able
Table 2: Values and standard deviations of the constrained orbital parameters for KIC 4480321. Note that from hereon, for all similar tables, $T_{0}$ is expressed in Hel. JD - 2,400,000. and rms indicates the standard deviation of the squared residuals.

\begin{tabular}{|c|c|c|}
\hline \multicolumn{3}{|c|}{ Orbital solution A-B } \\
\hline Orbital parameter & Value & Std. dev. \\
\hline$P(\mathrm{~d})$ & 9.16585 & 0.00015 \\
\hline$T_{0}(\mathrm{Hel} . \mathrm{JD})$ & 58503.065 & 0.015 \\
\hline$e$ & 0.07894 & 0.00097 \\
\hline$\omega\left(^{\circ}\right)$ & 351.1 & 0.6 \\
\hline$V_{\gamma}\left(\mathrm{km} \mathrm{s}^{-1}\right)$ & (var.) & \\
\hline$a_{\mathrm{A}} \sin i(\mathrm{au})$ & 0.04779 & 0.00006 \\
\hline$a_{\mathrm{B}} \sin i(\mathrm{au})$ & 0.04876 & 0.00006 \\
\hline$K_{\mathrm{A}}\left(\mathrm{km} \mathrm{s}^{-1}\right)$ & 56.90 & 0.07 \\
\hline$K_{\mathrm{B}}\left(\mathrm{km} \mathrm{s}^{-1}\right)$ & 58.05 & 0.07 \\
\hline$M_{\mathrm{A}} \sin ^{3} i\left(\mathrm{M}_{\odot}\right)$ & 0.7216 & 0.0019 \\
\hline$M_{\mathrm{B}} \sin ^{3} i\left(\mathrm{M}_{\odot}\right)$ & 0.7073 & 0.0019 \\
\hline$q_{\text {in }}$ & 0.980 & 0.002 \\
\hline$r m s_{\mathrm{A}}\left(\mathrm{km} \mathrm{s}^{-1}\right)$ & 1.486 & \\
\hline$r m s_{\mathrm{B}}\left(\mathrm{km} \mathrm{s}^{-1}\right)$ & 0.455 & \\
\hline \multicolumn{3}{|c|}{ Orbital solution AB-C } \\
\hline Orbital parameter & Value & Std. dev. \\
\hline$P(\mathrm{~d})$ & 2381. & 5.9 \\
\hline$T_{0}$ (Hel. JD) & 59082. & 13. \\
\hline$e$ & 0.140 & 0.006 \\
\hline$\omega\left(^{\circ}\right)$ & 197.9 & 1.8 \\
\hline$V_{\gamma}\left(\mathrm{km} \mathrm{s}^{-1}\right)$ & -19.97 & 0.04 \\
\hline$a_{\mathrm{AB}} \sin i_{\mathrm{out}}(\mathrm{au})$ & 2.232 & 0.013 \\
\hline$a_{\mathrm{C}} \sin i_{\text {out }}(\mathrm{au})$ & 3.293 & 0.076 \\
\hline$a_{\mathrm{TD}_{\mathrm{C}}} / c \sin i_{\text {out }}(\mathrm{d})$ & 0.0190 & 0.0004 \\
\hline$K_{\mathrm{AB}}\left(\mathrm{km} \mathrm{s}^{-1}\right)$ & 10.30 & 0.07 \\
\hline$K_{\mathrm{C}}\left(\mathrm{km} \mathrm{s}^{-1}\right)$ & 15.20 & 0.35 \\
\hline$M_{\mathrm{AB}} \sin ^{3} i_{\text {out }}\left(\mathrm{M}_{\odot}\right)$ & 2.36 & 0.12 \\
\hline$M_{\mathrm{C}} \sin ^{3} i_{\text {out }}\left(\mathrm{M}_{\odot}\right)$ & 1.60 & 0.05 \\
\hline$q_{\text {out }}$ & 0.678 & 0.016 \\
\hline$r m s_{\mathrm{C}}\left(\mathrm{km} \mathrm{s}^{-1}\right)$ & 4.689 & \\
\hline$r m s_{\mathrm{TD}}(\mathrm{d})$ & 0.0012 & \\
\hline
\end{tabular}

to determine a (much) improved mass ratio for the outer system and to provide (more) reliable values of the three minimum component masses. From the minimum total mass of the A-B system derived for each orbit, we get $\sin i=0.846( \pm 0.028)$ $\sin i_{\text {out }}$, from which we obtain the condition $i<58^{\circ}$ for the inner orbit. We may furthermore attribute the $\delta$ Scuti pulsations to the more massive component $\mathrm{C}$ (the frequencies showing phase shifts are all located in the $p$-mode region). In summary, KIC 4480321 is a hierarchical triple system with $q_{\text {in }}=M_{\mathrm{B}} / M_{\mathrm{A}}$ $=0.98$ and $q_{\text {out }}=M_{\mathrm{C}} / M_{\mathrm{AB}}=0.68$.

\subsection{The triple system KIC 5219533}

KIC 5219533 was classified as triple (SB3) with an inner pair of Am-like stars and with orbital periods of 31.919 and $1615 \mathrm{~d}$ (Sect. 2.2). For this system, we also detected long-term variations of the TDs in agreement with the long-term change observed in the RVs (Paper I, fig. 24). We performed a simultaneous least-squares fitting of the updated component RVs and the mean TDs with their respective uncertainties, and obtained the best fit in the sense of $\min \chi^{2}$ with the TDs assigned to 


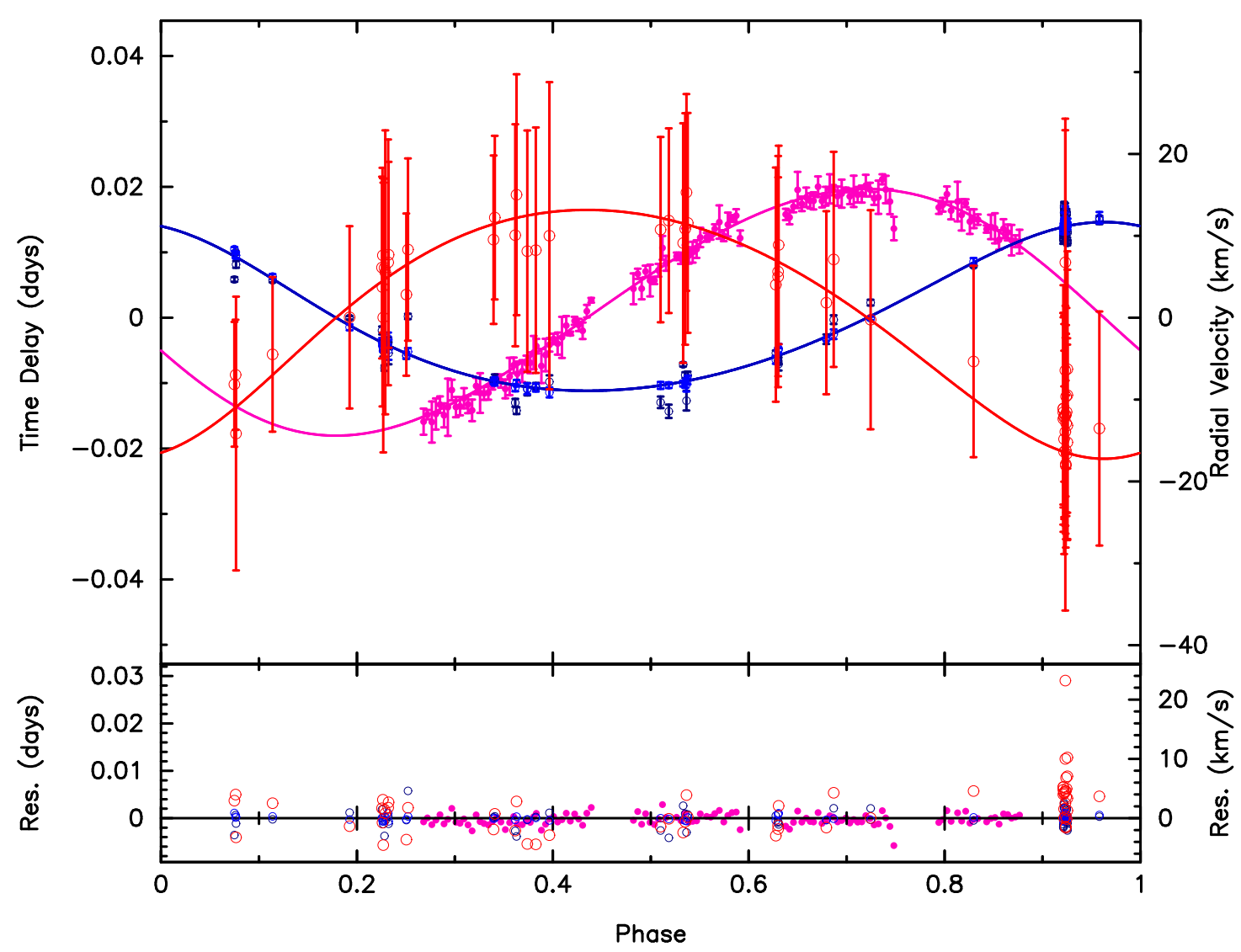

Fig. 1: Data and outer orbital solution for KIC 4480321. The TDs and RVs (resp. filled pink and unfilled red symbols for component $\mathrm{C}$; blue symbols for the centre of mass of components A and B) are overlaid with the AB-C orbital solution (solid lines). The residuals are shown in the bottom panel.

the outer companion (component C). Since this component is heavily diluted in the composite spectrum, its RVs are not well-determined. However, a modelling of the combined data allows to derive full orbital solutions. The new parameters of the inner and outer orbital solutions are presented in Table 3. Fig. 2 illustrates the quality of the fit as well as both solutions. The mean residuals of the component RVs stay below $1 \mathrm{~km} \mathrm{~s}^{-1}$. The largest residuals are found near the nodal passage (both components have similar RVs). The orbital solution of the A-B pair is in good agreement with the one proposed by Catanzaro et al. (2019a), though our semi-major axes are slightly larger (and our component masses a bit smaller) than theirs. Indeed, their Figs. 3 and 4 show that several HERMES RVs (open symbols) lie a bit off from their final solution. The overall gain in accuracy for most parameters of the inner orbital solution is a factor of 5, while the orbital period is 10 times more accurate. We cannot compare the mean RV residuals since these were not displayed. In Fig. 2 (right), the TDs and the RVs are plotted together with the revised AB-C orbital solution. We thus determined the semi-axis major of component $\mathrm{C}$ and the minimum mass of the A-B pair for the first time. The gain is largest for the outer orbit since the accuracy on the parameters improved with a mean factor of 10 while the orbital period is about 30 times more accurate compared to Catanzaro et al. (2019a). In particular, we obtained the minimum component masses $M_{\mathrm{A}} \sin ^{3} i=1.336 \pm 0.005, M_{\mathrm{B}} \sin ^{3} i=1.293 \pm 0.004$ and $M_{\mathrm{C}} \sin ^{3} i_{\text {out }}=1.47 \pm 0.02 \mathrm{M}_{\odot}$. From the minimum total mass of the A-B system derived for each orbit leading to the condition $\sin i_{\text {out }}=0.996( \pm 0.009) \sin i$, we infer that both orbits are very close to coplanarity. In summary, KIC 5219533 is a hierarchical, probably coplanar system with $q_{\text {in }}=0.97$ and $q_{\text {out }}=0.57$. We furthermore established that the $\delta$ Scuti pulsations are linked to the more massive, faster rotating star in the system.

\subsection{KIC 8975515}

This star was recognized as SB2 from our multi-epoch study, and also shows long-term variations of the TDs coherent with the variations found in the RVs (Paper I, fig. 24). The system consists of late A-type stars with one fast spinning (component A) and one (apparently) slowly spinning star (component B). In Paper I, we derived an orbital solution of type SB1 based on the RVs of component B only $\left(P_{\text {orb }} \sim 1600 \mathrm{~d}\right)$. We first derived a pure RV-based orbital solution (using the RVs of both components). Unfortunately, the extreme uncertainties on the RVs of the fast spinning component did not allow to constrain its RV amplitude. This is obvious in Fig. 3 (left) which illustrates the best-fit orbital solution with a period of $1581 \mathrm{~d}$. Subsequently, we performed two simultaneous (RV+TD) analyses, by associating the TDs with each set of RVs in turn, and found an optimum fit when the TDs were assigned to component A.

We determined an orbital period of $1603.4 \pm 9.3 \mathrm{~d}$. The new orbital parameters and their derived fundamental properties are listed in Table 4. The residuals show the very good agreement between both data types, with small mean residuals for the TDs and RVs of component B (the slower rotator), and a large (non-zero) mean residual for component A (the faster rotator). 

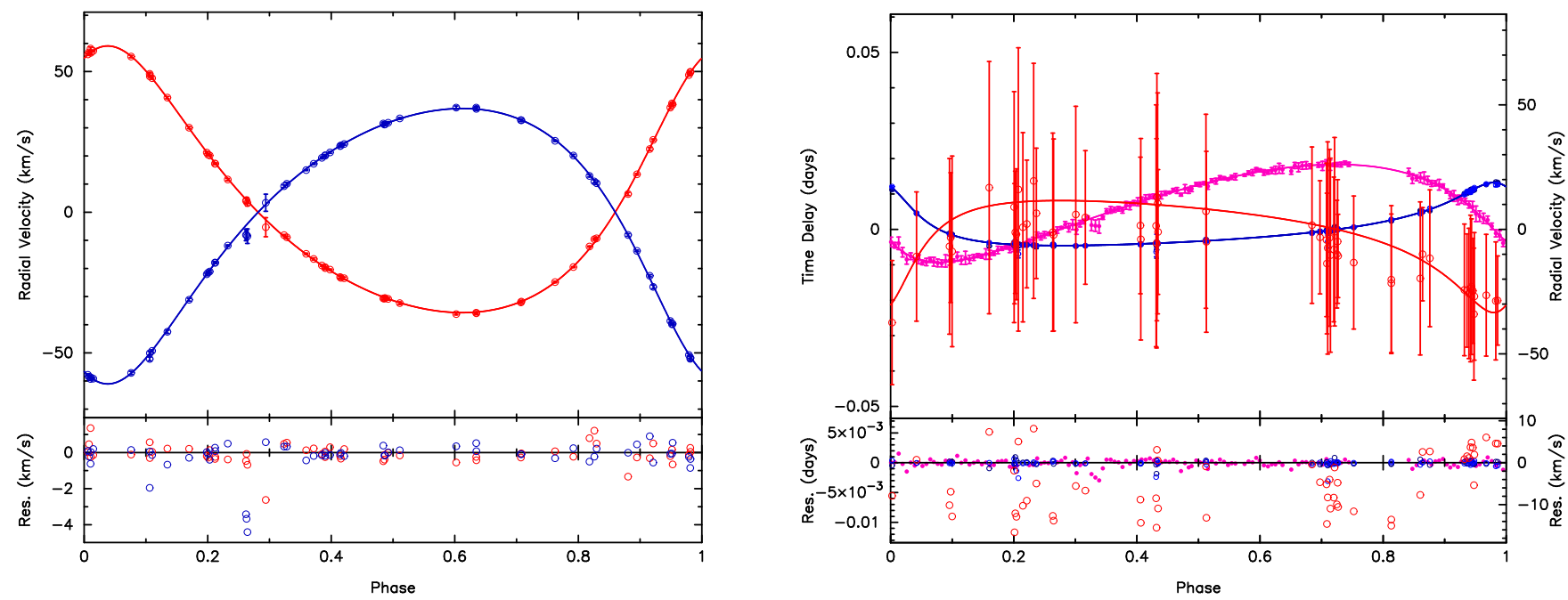

Fig. 2: Data and orbital solutions for KIC 5219533. Left: Inner orbit based on the HERMES RVs. The RVs (red symbols for component A; blue symbols for component B) are overlaid with the A-B orbital solution (solid lines). Right: Outer orbit based on the HERMES RVs and the TDs from the Kepler photometry. The TDs and RVs (resp. filled pink and unfilled ed symbols for component C; blue symbols for the centre of mass of components A and B) are overlaid with the AB-C orbital solution (solid lines). The residuals are shown in the bottom panels.

Table 3: Values and standard deviations of the constrained orbital parameters for KIC 5219533.

\begin{tabular}{|c|c|c|}
\hline \multicolumn{3}{|c|}{ Orbital solution A-B } \\
\hline Orbital parameter & Value & Std. dev. \\
\hline$P(\mathrm{~d})$ & 31.91763 & 0.00022 \\
\hline$T_{0}(\mathrm{Hel} . \mathrm{JD})$ & 57467.41 & 0.02 \\
\hline$e$ & 0.271 & 0.001 \\
\hline$\omega\left(^{\circ}\right)$ & 335.3 & 0.2 \\
\hline$V_{\gamma}\left(\mathrm{km} \mathrm{s}^{-1}\right)$ & (var.) & \\
\hline$a_{\mathrm{A}} \sin i(\mathrm{au})$ & 0.13369 & 0.00019 \\
\hline$a_{\mathrm{B}} \sin i(\mathrm{au})$ & 0.13810 & 0.00023 \\
\hline$K_{\mathrm{A}}\left(\mathrm{km} \mathrm{s}^{-1}\right)$ & 47.33 & 0.07 \\
\hline$K_{\mathrm{B}}\left(\mathrm{km} \mathrm{s}^{-1}\right)$ & 48.90 & 0.08 \\
\hline$M_{\mathrm{A}} \sin ^{3} i\left(\mathrm{M}_{\odot}\right)$ & 1.336 & 0.005 \\
\hline$M_{\mathrm{B}} \sin ^{3} i\left(\mathrm{M}_{\odot}\right)$ & 1.293 & 0.004 \\
\hline$q_{\text {in }}$ & 0.968 & 0.002 \\
\hline$r m s_{\mathrm{A}}\left(\mathrm{km} \mathrm{s}^{-1}\right)$ & 0.550 & \\
\hline$r m s_{\mathrm{B}}\left(\mathrm{km} \mathrm{s}^{-1}\right)$ & 0.833 & \\
\hline \multicolumn{3}{|c|}{ Orbital solution AB-C } \\
\hline Orbital parameter & Value & Std. dev. \\
\hline$P(\mathrm{~d})$ & 1603.5 & 1.7 \\
\hline$T_{0}$ (Hel. JD) & 55212.9 & 3.2 \\
\hline$e$ & 0.564 & 0.004 \\
\hline$\omega\left(^{\circ}\right)$ & 210.6 & 0.5 \\
\hline$V_{\gamma}\left(\mathrm{km} \mathrm{s}^{-1}\right)$ & 10.70 & 0.04 \\
\hline$a_{\mathrm{AB}} \sin i_{\text {out }}(\mathrm{au})$ & 1.542 & 0.008 \\
\hline$a_{\mathrm{C}} \sin i_{\text {out }}(\mathrm{au})$ & 2.74 & 0.03 \\
\hline$a_{\mathrm{TD}_{\mathrm{C}}} / c \sin i_{\text {out }}(\mathrm{d})$ & 0.0158 & 0.0002 \\
\hline$K_{\mathrm{AB}}\left(\mathrm{km} \mathrm{s}^{-1}\right)$ & 12.67 & 0.08 \\
\hline$K_{\mathrm{C}}\left(\mathrm{km} \mathrm{s}^{-1}\right)$ & 22.49 & 0.27 \\
\hline$M_{\mathrm{AB}} \sin ^{3} i_{\text {out }}\left(\mathrm{M}_{\odot}\right)$ & 2.60 & 0.07 \\
\hline$M_{\mathrm{C}} \sin ^{3} i_{\text {out }}\left(\mathrm{M}_{\odot}\right)$ & 1.47 & 0.02 \\
\hline$q_{\text {out }}$ & 0.565 & 0.006 \\
\hline$r m s_{\mathrm{C}}\left(\mathrm{km} \mathrm{s}^{-1}\right)$ & 8.294 & \\
\hline$r m s_{\mathrm{TD}}(\mathrm{d})$ & 0.0006 & \\
\hline
\end{tabular}

Table 4: Values and standard deviations of the constrained orbital parameters for KIC 8975515.

\begin{tabular}{lcl}
\hline \hline \multicolumn{3}{c}{ Orbital solution A-B } \\
\hline Orbital parameter & Value & Std. dev. \\
\hline$P(\mathrm{~d})$ & 1603.4 & 9.3 \\
$T_{0}(\mathrm{Hel} . \mathrm{JD})$ & 58690. & 19. \\
$e$ & 0.408 & 0.015 \\
$\omega\left({ }^{\circ}\right)$ & 172.5 & 2.6 \\
$V_{\gamma}\left(\mathrm{km} \mathrm{s}^{-1}\right)$ & -20.63 & 0.14 \\
$a_{\mathrm{A}} \sin i(\mathrm{au})$ & 0.3988 & 0.0053 \\
$a_{\mathrm{B}} \sin i(\mathrm{au})$ & 0.483 & 0.028 \\
\hline$a_{\mathrm{TD}} / c \sin i(\mathrm{~d})$ & 0.00230 & 0.00003 \\
$K_{\mathrm{A}}\left(\mathrm{km} \mathrm{s}^{-1}\right)$ & 2.96 & 0.05 \\
$K_{\mathrm{B}}\left(\mathrm{km} \mathrm{s}^{-1}\right)$ & 3.59 & 0.21 \\
$M_{\mathrm{A}} \sin ^{3} i\left(\mathrm{M}_{\odot}\right)$ & 0.0195 & 0.0024 \\
$M_{\mathrm{B}} \sin ^{3} i\left(\mathrm{M}_{\odot}\right)$ & 0.0161 & 0.0011 \\
$q$ & 0.83 & 0.05 \\
\hline$r m s_{\mathrm{A}}\left(\mathrm{km} \mathrm{s}^{-1}\right)$ & 2.597 & \\
$r m s_{\mathrm{B}}\left(\mathrm{km} \mathrm{s}^{-1}\right)$ & 0.237 & \\
$r m s_{\mathrm{TD}}(\mathrm{days})$ & 0.00013 \\
\hline
\end{tabular}

The orbital solution is illustrated in Fig. 3 (right). In this case, the mass ratio $(q=0.83 \pm 0.05)$ is well-determined thanks to the high accuracy of the TDs (whereas the corresponding component RVs show a very large scatter). The present method allowed us to derive information which was otherwise impossible to obtain with a pure RV-based solution. Another conclusion is that the faster rotating component exhibits the $\delta$ Scuti pulsations. This may also explain why the RV residuals of component A are found to be systematically off from the final solution. From the parameters displayed in Table 4 , we obtain the minimum masses, $M_{\mathrm{A}} \sin ^{3} i=0.0195 \pm 0.0024$ and $M_{\mathrm{B}} \sin ^{3} i=0.0161 \pm$ $0.0011 \mathrm{M}_{\odot}$. Assuming that either one of the components has a typical mass of $\sim 2 \mathrm{M}_{\odot}$, we expect that the system is seen under a low inclination angle $\left(i \sim 12^{\circ}\right)$. 

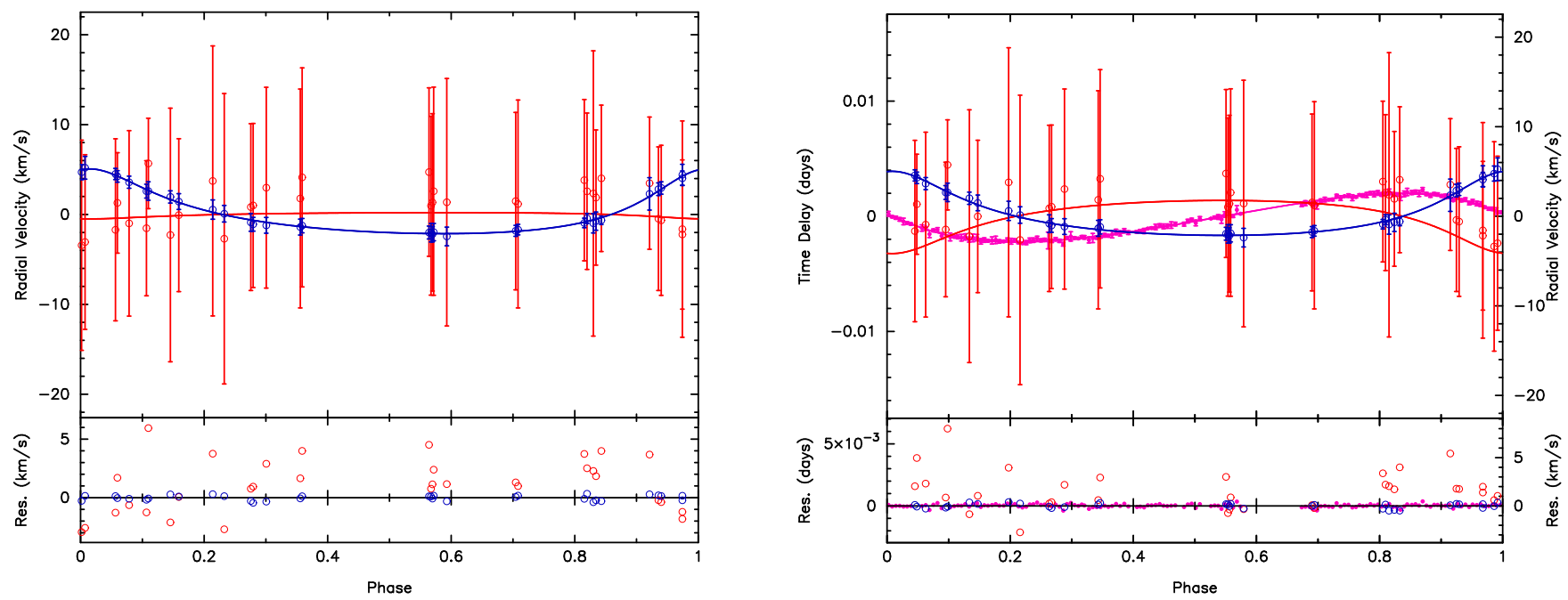

Fig. 3: Data and orbital solution for KIC 8975515. Left: Orbit (nr 1) based on the HERMES RVs. The RVs (red symbols for component A; blue symbols for component B) are overlaid with a preliminary orbital solution of type SB2, however without much constraint on the amplitude $\mathrm{K}_{A}$. Right: Orbit (nr 2) based on the HERMES RVs and the TDs from the Kepler photometry. The TDs and RVs (resp. filled pink and unfilled red symbols for component A; blue symbols for component B) are overlaid with the A-B orbital solution (solid lines). The residuals are shown in the bottom panels.

\subsection{KIC 9775454}

KIC 9775454 was classified as a long-period SB1 (Sect. 2.4). It was shown to display long-term variations of the TDs in agreement with the orbital variations of the RVs (this concerns also the dominant frequency located at $4.161 \mathrm{~d}^{-1}$, cf. Paper I). Murphy et al. (2018) used the HERMES RVs of the broad-lined primary (component A) and derived an orbital solution with a period of $\sim 1700$ days from their combined (RV+TD) analysis. Being much fainter, component B was not directly detected in the high-resolution spectra. Nevertheless, we managed to find an indication of a cool companion by computing CCFs of the residual spectra (after proper subtraction of an adequate synthetic spectrum to remove the contribution of the primary) using a mask of spectral type K0. The "residual" CCFs were computed for the spectral range [5100 - 5700] $\AA$, and revealed a shallow peak that reflects the contribution of the companion and displays Doppler shifts (Fig. 5). In this way, we were able to extract additional RV data which behave in anti-phase with those of component A (Fig. 4). We roughly estimated their uncertainties from the widths of the associated spectral lines. Thus, we can classify KIC 9775454 as an SB2. We next performed a simultaneous (RV+TD) analysis, by fitting the component RVs together with the TDs assigned to the broad-lined primary (component A). The new orbital solution is illustrated by Fig. 4. The RV residuals of component A and the TD residuals are within expectation while the RV residuals of component B are systematically large. This can be explained by the noisy "residual" CCFs associated to the difficulty of identifying the lines of the cool component in the spectra. The orbital parameters and derived fundamental properties are listed in Table 5. These parameters agree very well with the values proposed by Murphy et al. (2018). By treating this system as an SB2 and using the TDs, we determined for the first time a reasonable value of the mass ratio $(q=0.42 \pm 0.02)$. In this special case where the TDs are linked with both the low- and the high-frequency regions, the more massive primary component is very likely the hybrid pulsator.

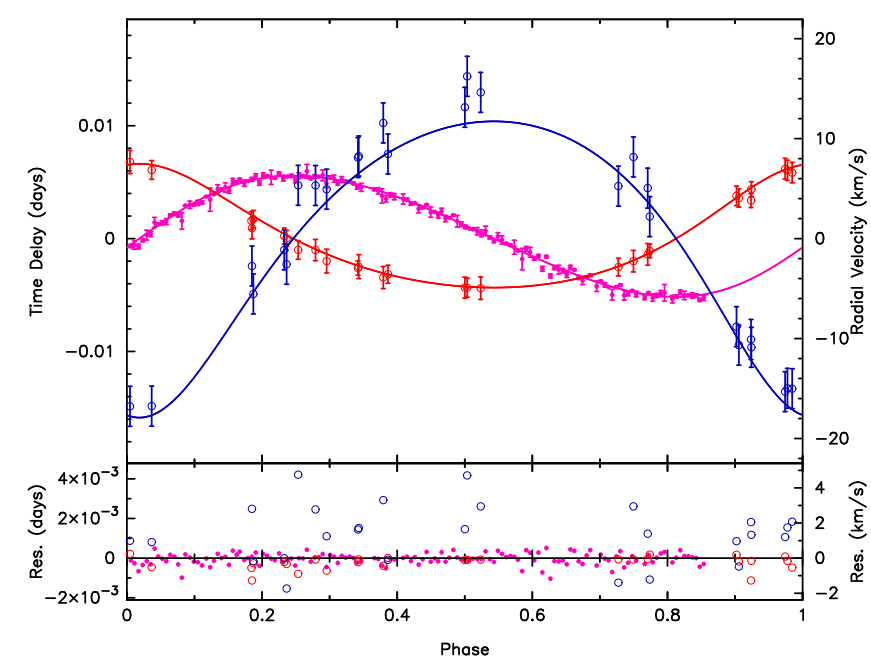

Fig. 4: Data and orbital solution for KIC 9775454. The TDs (filled pink symbols) and the RVs (unfilled red symbols for component A; blue symbols for component B) are overlaid with the A-B orbital solution (solid lines). The residuals are shown in the bottom panel.

\section{Search for regular frequency patterns}

In this section, we investigate the distributions of the frequency differences (aka spacings) that occur in the high-frequency regime of the Fourier transforms of each system. The aim is to identify the (most) frequently occurring frequency spacings for each object, and to verify whether some of these spacings might be related to the orbital or the rotational periods. In the case of an orbital or a rotational origin of the low frequencies (e.g. due to modulations in the light curve caused by the tidal deformations or by the presence of spots or other surface inhomogeneities e.g. MOBSTER (Sikora et al. 2019) or caused by the mechanism of tidal excitation e.g. KIC 4142768 (Guo et al. 2019), we may expect to detect a significant number of harmonics of the corresponding main frequencies. In the case of 


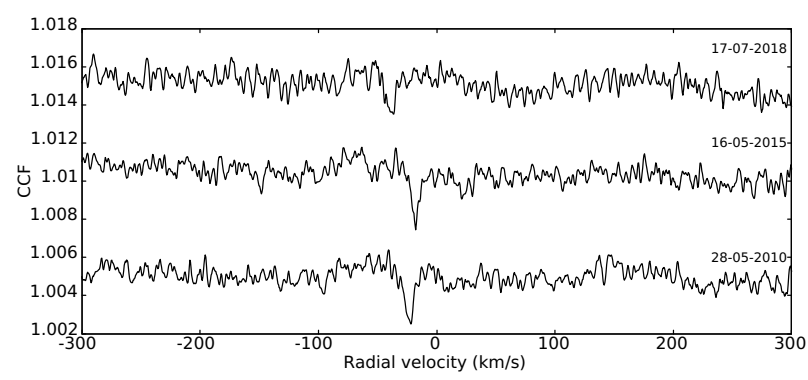

Fig. 5: CCFs of the residual spectra of KIC 9775454 computed with a mask of type K0 for three different dates.

Table 5: Values and standard deviations of the constrained parameters of the orbital solution for KIC 9775454.

\begin{tabular}{lcc}
\hline \hline \multicolumn{3}{c}{ Orbital solution A-B } \\
\hline Orbital parameter & Value & Std. dev. \\
\hline$P(\mathrm{~d})$ & 1706.8 & 6.4 \\
$T_{0}(\mathrm{Hel} . \mathrm{JD})$ & 56655. & 10. \\
$e$ & 0.212 & 0.008 \\
$\omega\left(^{\circ}\right)$ & 349.6 & 1.9 \\
$V_{\gamma}\left(\mathrm{km} \mathrm{s}^{-1}\right)$ & -22.15 & 0.16 \\
$a_{\mathrm{A}} \sin i(\mathrm{au})$ & 0.949 & 0.017 \\
$a_{\mathrm{B}} \sin i(\mathrm{au})$ & 2.27 & 0.09 \\
\hline$a_{\mathrm{TD}} / c \sin i(\mathrm{~d})$ & 0.00548 & 0.00010 \\
$K_{\mathrm{A}}\left(\mathrm{km} \mathrm{s}^{-1}\right)$ & 6.19 & 0.12 \\
$K_{\mathrm{B}}\left(\mathrm{km} \mathrm{s}^{-1}\right)$ & 14.82 & 0.62 \\
$M_{\mathrm{A}} \sin ^{3} i\left(\mathrm{M}_{\odot}\right)$ & 1.08 & 0.11 \\
$M_{\mathrm{B}} \sin ^{3} i\left(\mathrm{M}_{\odot}\right)$ & 0.45 & 0.03 \\
$q$ & 0.42 & 0.02 \\
\hline$r m s_{\mathrm{A}}\left(\mathrm{km} \mathrm{s}^{-1}\right)$ & 0.471 & \\
$r m s_{\mathrm{B}}\left(\mathrm{km} \mathrm{s}^{-1}\right)$ & 2.150 & \\
$r m s_{\mathrm{TD}}(\mathrm{d})$ & 0.00028 \\
\hline
\end{tabular}

a tidal or rotational splitting of the high frequencies associated to the rapid pulsations (e.g. of tidal splitting of $p$-modes in KIC 6048106 and U Gru, respectively, Samadi Ghadim et al. 2018; Bowman et al. 2019), we may expect to find regular (also non-harmonic) frequency patterns whose spacing values will point at those frequencies.

We used the detrending algorithm of Lightkurve v1.9 to detrend the Kepler light curves of the previously discussed systems (Barentsen \& Lightkurve Collaboration 2020), and computed the periodograms with the Lomb-Scargle technique (Fig. 6). For each system, we analysed the frequency interval of the periodogram where most of the power is located. For KIC 4480321, this corresponds to the $[12.5,24.7] \mathrm{d}^{-1}$ interval; for KIC 5219533, this is the $[6,22.5] \mathrm{d}^{-1}$ inter$\mathrm{val}$; for KIC 8975515 , the $[12.5,17.5] \mathrm{d}^{-1}$ interval, and for KIC 9775454, the $[5.7,22.5] \mathrm{d}^{-1}$ interval. We then computed the spacings between all possible pairs of significant frequencies while considering the limiting frequency resolution of the Kepler long-cadence data sets, and counted the number of individual occurrences (as in Samadi Ghadim et al.2018).

We next discuss the density distributions of the frequency spacings derived from the Kepler-based periodograms for the four systems with the improved orbits. The density plots of the frequency spacings were produced from the counts adopting a bin size of $0.05 \mathrm{~d}^{-1}$ in each case. In Fig. 7) we show the density plots of the spacings for KIC 4480321, 5219533, 8975515 and KIC 9775454 in the restricted frequency range $[0-4] \mathrm{d}^{-1}$, i.e. the interval where the highest occurrences can be found. A general observation is that the distributions of the frequency spacings in the high-frequency regime of the four systems show large differences between them. Also, a high(er) peak in the distributions (or a secondary maximum) of individual spacings is generally located in the first bin. This is due to the fact that this bin also contains the smallest possible spacing which corresponds to the frequency resolution of $0.00068 \mathrm{~d}^{-1}$ in the Kepler long-cadence data.

From Fig. 7, we derived the following findings:

- Both KIC 4480321 and KIC 5219533 present a dense and continuous distribution of frequency spacings with several (almost) equal local maxima. There is power everywhere, also in the first bin. The close binaries of these triple systems have orbital frequencies of respectively $0.11 \mathrm{~d}^{-1}$ and $0.03 \mathrm{~d}^{-1}$, which means that in the case of KIC 4480321, the bin size is small enough to draw a firm conclusion with respect to tidal splitting. In the case of the remaining binary systems, the orbital periods are of order of several years and too large to be resolved in the periodograms. In the case of KIC 4480321, the highest occurrences occur at the spacings of $0.35,1.63$ (highest), and $3.33 \mathrm{~d}^{-1}$. The latter spacing shows a harmonic relation with $1.63 \mathrm{~d}^{-1}$ (ratio of almost 2 ). This interdependence between two frequent spacings could be very well explained by rotation. Also, Li et al. (2020b) derived $0.0070 \pm 0.0007 \mathrm{~d}^{-1}$ for the near-core rotation rate from a modelling of the slope-period relation based on the regular period spacings in the $g$-mode region. Such a fast rotation rate stands in sharp contrast with the the abovelisted most frequent spacings if we consider them as potential surface rotation rates for component $\mathrm{C}$ (the fast-spinning component). On the other hand, we find no peak occurring near or at the orbital frequency value of $0.11 \mathrm{~d}^{-1}$. We conclude that the orbital frequency of the inner binary system does not affect the pulsation frequencies in the $p$-mode region. This is perfectly consistent with the conclusion that component $\mathrm{C}$ is the $\delta$ Scuti pulsator.

- In the case of KIC 5219533, the most frequent spacings occur at the frequencies of $0.55,0.94$ (highest) and $2.32 \mathrm{~d}^{-1}$, (or the equally frequent spacings at 2.98 and $3.59 \mathrm{~d}^{-1}$ ). The frequent spacings of 0.55 and $0.94 \mathrm{~d}^{-1}$ could indicate the rotation period of component $\mathrm{C}$ (the fast-spinning component). We note that Li et al. (2020b) derived $0.59 \pm 0.01 \mathrm{~d}^{-1}$ for the near-core rotation rate based on their modelling. This seems to match the smallest value of the frequent spacings (i.e. $0.55 \mathrm{~d}^{-1}$ ). The orbital period of the inner binary is $\sim 32 \mathrm{~d}$, which implies that the corresponding frequency (at $0.03 \mathrm{~d}^{-1}$ ) would be located in the first bin. Thus, we cannot infer from this plot whether or not this frequency is affecting the higher pulsation frequencies. However, the most frequent individual spacing occurs at $0.0012 \mathrm{~d}^{-1}$, which is far away from the orbital frequency of the inner pair.

- KIC 8975515 presents an irregular distribution of unequal peaks, though of much lower density. The two highest occurrences (except for the first bin) occur at 2.10 (highest) and $3.76 \mathrm{~d}^{-1}$, closely followed by $1.06 \mathrm{~d}^{-1}$. Again, the ratio of $\sim$ 2 between two of the three most frequent spacings indicates that rotation might be the relevant mechanism to explain the presence of a frequent spacing and its harmonic. However, the frequency spacing of $1.66 \mathrm{~d}^{-1}$, which probably corresponds to the rotation frequency of the fast-rotating hybrid pulsating component in the system (Samadi-Ghadim et al. 2020), is the highest occurrence among the individual spacings. Li et al. (2020b) derived $1.85 \pm 0.01 \mathrm{~d}^{-1}$ for the near-core rotation rate. 

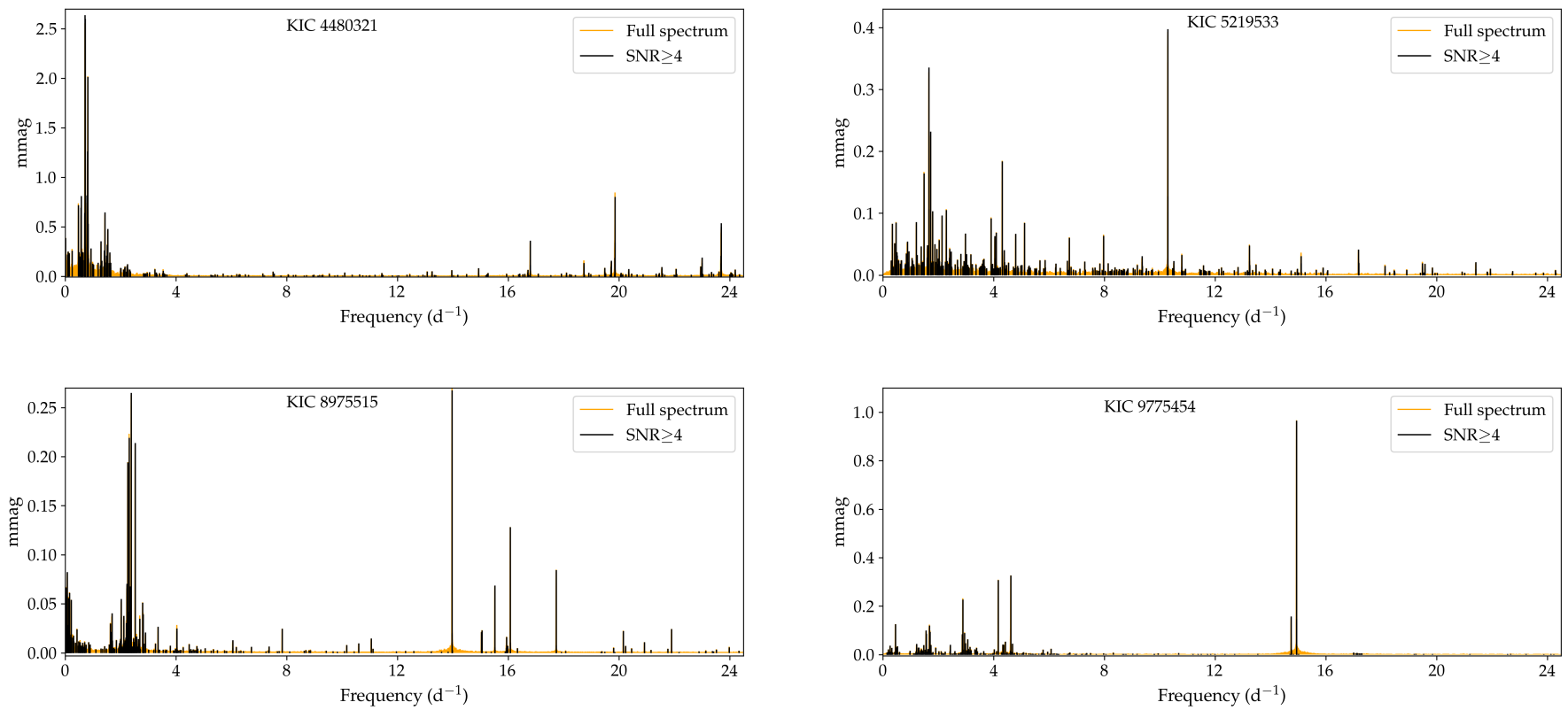

Fig. 6: Lomb-Scargle periodograms derived from the Kepler data for the systems KIC 4480321 (top left), 5219533 (top right), 8975515 (bottom left) and KIC 9775454 (bottom right). The frequencies plotted in orange illustrate the full spectrum across the range $[0-24.5] \mathrm{d}^{-1}$, while the frequencies overplotted in black have a signal-to-noise ratio higher than 4.

If this rate corresponds to the faster rotating component, the core versus surface rotation ratio would equal $1.11 \pm 0.01$, as expected from the general trend found by $\mathrm{Li}$ et al. (2020b). The frequent spacing of $1.06 \mathrm{~d}^{-1}$ might correspond to the more slowly-rotating $\delta$ Scuti component, although Samadi-Ghadim et al. (2020) proposed a rotation rate of $0.42 \mathrm{~d}^{-1}$. In this case, the orbital frequency is unresolved.

- KIC 9775454 shows a remarkably sparse distribution in frequency spacing. The two highest peaks (except for the first bin) occur at the spacings of 0.13 and $2.18 \mathrm{~d}^{-1}$ (highest). The latter and most frequent spacing is accompanied by a few nearby, almost equally frequent spacings located at 2.07, 2.26 and $2.35 \mathrm{~d}^{-1}$. This suggests that one of these frequency spacings is the rotational frequency of the hybrid pulsator (comp A) (in the case of $\sim 2 \mathrm{~d}^{-1}$, it is probably variable). If we consider the fact that the primary component has $v \sin i=65 \mathrm{~km} \mathrm{~s}^{-1}$, adopting a default radius of $1.8 \mathrm{R}_{\odot}$ corresponding to its $T_{\text {eff }}$ (Gorda \& Svechnikov 1998) and the frequency of $2.18 \mathrm{~d}^{-1}$ as the rotational frequency, implies $v_{\mathrm{eq}}=199 \mathrm{~km} \mathrm{~s}^{-1}$ and a surface inclination angle of $\sim 19^{\circ}$ as possible values. The alternative choice (with $0.13 \mathrm{~d}^{-1}$ ) would give an impossible value of $12 \mathrm{~km} \mathrm{~s}^{-1}$ for $v_{\mathrm{eq}}$. The orbital frequency is unresolved here as well.

\section{Summary and conclusions}

In the previous sections, we improved the orbital solutions for four spectroscopic systems from our sample of 49 A/F-type Kepler hybrid stars. We improved the orbital solutions for the following spectroscopic systems: KIC 4480321, 5219533, 8975515 and KIC 9775454 by considering the TDs as well as the RVs and performing a simultaneous modelling of both data types. This method allowed us to refine the parameters of all the long-period systems, in particular the outer orbits of the triple systems KIC 4480321 and KIC 5219533. We derived full-fledged SB2 orbital solutions for the long-period binary systems KIC 8975515 and KIC 9775454 and obtained reliable mass ratios for the first time. Furthermore, the applied methodology enabled us to identify the component with the faster $\delta$ Sct-type pulsations, since the TDs are generally linked with the higher frequencies.

For KIC 4480321, we concluded that the faster rotating and more massive outer component (comp C) exhibits the short-period $\delta$ Scuti pulsations. Component $\mathrm{C}$ was shown to have a minimum mass of $1.60 \pm 0.05 \mathrm{M}_{\odot}$, which can be used as a constraint for asteroseismic modelling. For KIC 5219533, we significantly improved the accuracy of the orbital solutions described by Catanzaro et al. (2019a). We also showed that both orbits are very probably coplanar and that the $\delta$ Scuti pulsator is the faster spinning and more massive outer component (comp C). For KIC 8975515, we derived an accurate mass ratio $(q=0.83 \pm 0.05)$ and established that the faster rotating component (comp A) exhibits $\delta$ Scuti-type pulsations (this concerns the hybrid pulsator). Furthermore, the binary is probably viewed under a low inclination angle $\left(\approx 12^{\circ}\right)$. The latter is also useful information in an asteroseismic context. For KIC 9775454, we determined a precise mass ratio for the first time $(q=0.42 \pm 0.02)$. We concluded that the more massive component of the system (comp A) is the hybrid pulsating star.

From our study of the normalized distributions of the frequency spacings in the high-frequency regime of the Fourier transforms, we find no firm evidence for the occurrence of tidal splitting among the frequent spacings of the triple systems with close (inner) companions. In the case of the long-period orbits (all systems), it is impossible to resolve the frequency multiplets based on the Kepler data only. On the other hand, due to the presence of some harmonic frequencies among the most frequent spacings, we propose the mechanism of rotational splitting for KIC 4480321 (with a plausible surface rotation rate of $1.63 \mathrm{~d}^{-1}$ ), KIC 5219533 (with a possible surface rotation 

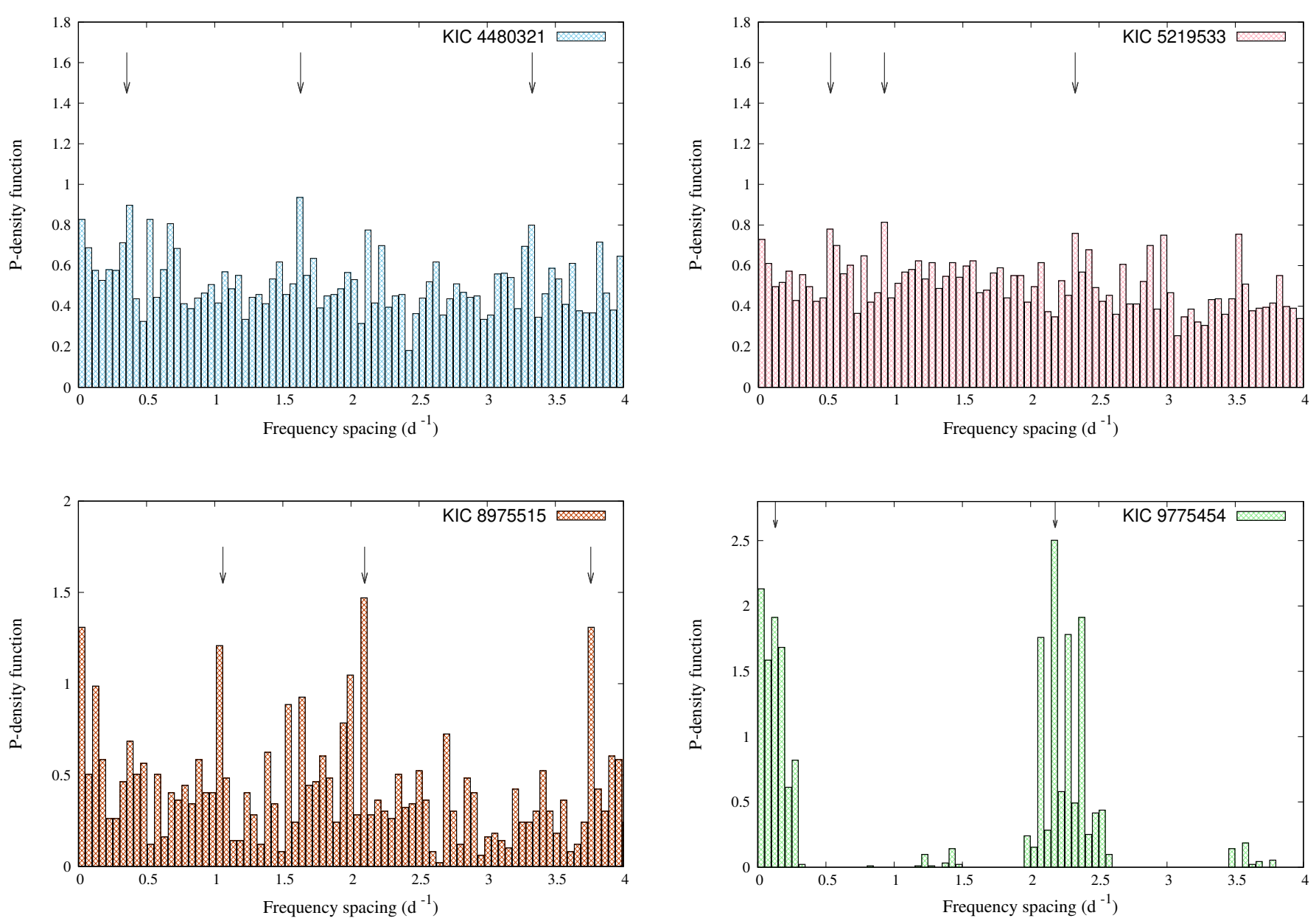

Fig. 7: Normalized density functions of the frequency spacings across the range $[0-4] \mathrm{d}^{-1}$ derived from the Kepler-based periodograms for the systems KIC 4480321 (top left), 5219533 (top right), 8975515 (bottom left) and KIC 9775454 (bottom right). The highest occurrences are marked by grey arrows.

rate of $0.55 \mathrm{~d}^{-1}$ ) and KIC 8975515 (with a possible surface rotation rate of $1.06 \mathrm{~d}^{-1}$ for the secondary component, whereas the primary rotates at the rate of $1.66 \mathrm{~d}^{-1}$ ). In the particular case of KIC 9775454, we propose a surface rotation rate of the order of $2 \mathrm{~d}^{-1}$, which appears as a bunch of closely spaced frequent spacings in the distribution and indicates a possibly variable nature. Detailed analyses of the pulsations in the low-frequency regime of the Fourier transforms of these systems, including pattern recognition and period spacings of the gravity (and sometimes Rossby) modes for the (rapidly rotating) genuine hybrid pulsators, can provide the near-core rotation rates and a confirmation of the proposed surface rotation rates (e.g., Van Reeth et al. 2016, Li et al. 2020ba).

\section{Acknowledgements}

The authors wish to thank the Kepler team for providing the high-quality data collected with the Kepler satellite mission. They furthermore thank the HERMES Consortium for enabling the production of the high-resolution ground-based spectra, and D. Bowman et al. for authorizing the use of the additional spectra of KIC 5219533. ÁS and ZsB acknowledge financial support of the Lendület Program LP2018-7/2019 of the Hungarian
Academy of Sciences. MS acknowledges the OV PPP PostdocMUNI grant with nr. CZ.02.2.69/0.0/0.0/16_027/0008360 and the MSMT Inter Transfer program LTT20015. ZsB acknowledges the support provided by the National Research, Development and Innovation Fund of Hungary, financed under the $\mathrm{PD}_{17}$ funding scheme (project PD-123910) and by the János Bolyai Research Scholarship of the Hungarian Academy of Sciences. HL acknowledges support from the grant DFG with nr. LE1102/3-1. ASG acknowledges financial support received from the ALMA-CONICYT grant with nr. 31170029. We thank the referee, Prof. H. Shibahashi, for detailed comments and constructive criticism. We are also grateful for support received from the Belgo-Indian Network for Astronomy \& Astrophysics (BINA-1 and BINA-2).

\section{References}

Balona, L. A., Daszyńska-Daszkiewicz, J., \& Pamyatnykh, A. A. 2015, MNRAS, 452, 3073

Barentsen, G. \& Lightkurve Collaboration. 2020, in American Astronomical Society Meeting Abstracts, American Astronomical Society Meeting Abstracts, 409.04

Bowman, D. M. 2017, Amplitude Modulation of Pulsation Modes in Delta Scuti Stars (Springer International Publishing)

Bowman, D. M., Johnston, C., Tkachenko, A., et al. 2019, ApJ, 883, L26 
Castelli, F. \& Kurucz, R. L. 2003, in IAU Symposium, Vol. 210, Modelling of Stellar Atmospheres, ed. N. Piskunov, W. W. Weiss, \& D. F. Gray, A20

Catanzaro, G., Gangi, M., Giarrusso, M., Munari, M., \& Leone, F. 2019a, MNRAS, 487, 919

Catanzaro, G., Gangi, M., Giarrusso, M., Munari, M., \& Leone, F. 2019b, MNRAS, 488,480

Fauvaud, S., Sareyan, J. P., Ribas, I., et al. 2010, A\&A, 515, A39

Gaia Collaboration. 2018, VizieR Online Data Catalog, I/345

Gorda, S. Y. \& Svechnikov, M. A. 1998, Astronomy Reports, 42, 793

Grigahcène, A., Antoci, V., Balona, L., et al. 2010, ApJ, 713, L192

Guo, Z., Fuller, J., Shporer, A., et al. 2019, ApJ, 885, 46

Hubeny, I. \& Lanz, T. 1995, ApJ, 439, 875

Irwin, J. B. 1952, ApJ, 116, 211

Lampens, P., Frémat, Y., Vermeylen, L., et al. 2018, A\&A, 610, A17

Li, G., Bedding, T. R., Murphy, S. J., et al. 2019, MNRAS, 482, 1757

Li, G., Guo, Z., Fuller, J., et al. 2020a, MNRAS, 497, 4363

Li, G., Van Reeth, T., Bedding, T. R., et al. 2020b, MNRAS, 491, 3586

Moffett, T. J., Barnes, Thomas G., I., Fekel, Francis C., J., Jefferys, W. H., \& Achtermann, J. M. 1988, AJ, 95, 1534

Murphy, S. J., Bedding, T. R., Shibahashi, H., Kurtz, D. W., \& Kjeldsen, H. 2014, MNRAS, 441, 2515

Murphy, S. J., Moe, M., Kurtz, D. W., et al. 2018, MNRAS, 474, 4322

Murphy, S. J. \& Shibahashi, H. 2015, MNRAS, 450, 4475

Murphy, S. J., Shibahashi, H., \& Bedding, T. R. 2016, MNRAS, 461, 4215

Nielsen, M. B., Gizon, L., Schunker, H., \& Karoff, C. 2013, A\&A, 557, L10

Pribulla, T., Chochol, D., Tremko, J., \& Kreiner, J. M. 2005, in Astronomical Society of the Pacific Conference Series, Vol. 335, The Light-Time Effect in Astrophysics: Causes and cures of the O-C diagram, ed. C. Sterken, 103

Raskin, G., van Winckel, H., Hensberge, H., et al. 2011, A\&A, 526, A69

Renson, P. \& Manfroid, J. 2009, A\&A, 498, 961

Samadi Ghadim, A., Lampens, P., \& Jassur, D. M. 2018, Acta Astron., 68, 425

Samadi-Ghadim, A., Lampens, P., Jassur, D. M., \& Jofré, P. 2020, A\&A, 638, A57

Shibahashi, H. \& Kurtz, D. W. 2012, MNRAS, 422, 738

Sikora, J., David-Uraz, A., Chowdhury, S., et al. 2019, MNRAS, 487, 4695

Uytterhoeven, K., Moya, A., Grigahcène, A., et al. 2011, A\&A, 534, A125

Van Reeth, T., Tkachenko, A., \& Aerts, C. 2016, A\&A, 593, A120 